\title{
ANALYSE DE LA POPLITIQUE HYDRIQUE EN ALGERIE DEPUIS L'INDEPENDENCE
}

\begin{abstract}
:
Like the other countries in the region, given its position in an arid and semi-arid climate, Algeria has suffered and will suffer from any scarcity of the indispensable resource for life on earth, which is why the authorities opted through different planes (three-year and five-year) various programs of investment in the water sector that have reached $\$ 22.5$ billion until 2008 with the estimate to reach 35.71 billion $\$$ in 2025.But yet to assess the effectiveness of these colossal sums and measure the return on investment, a value that is expressed in the form of Well-Being of the population and the impact of the resource on the socioeconomic development in terms of economic contribution.

Unrealistic goals are set during the development of water saving and modernization program as a unilateral decision, while history shows us that it is utopian visions, however these approaches are implemented by the allocation of huge sums without much guarantee or at least evaluate the effectiveness of programs developed and their impact on the socio-economic sphere and/or societal. As these amounts will not be helpful as governance is not assured with an effective strategy to be more realistic and to achieve the targeted goals.
\end{abstract}

\author{
Mohamed Sofiane CHAOUI \\ Pr. Azzedine BENTERKI \\ Université Constantine 2, Algérie \\ Nora VAN CAUWENBERGH \\ UNESCO-IHE, Delft, Pays-Bas
}

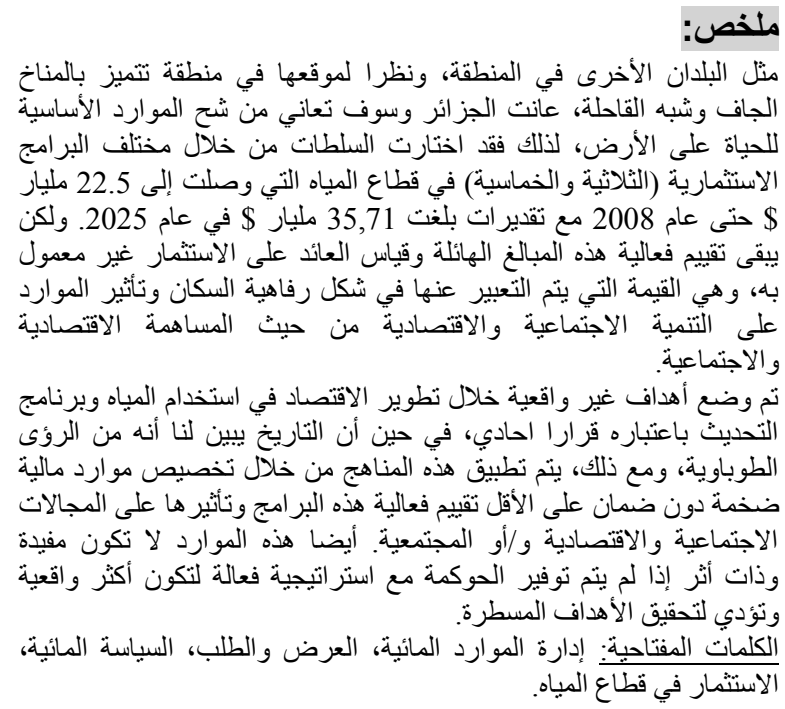

\section{Introduction :}

Considérée comme la denrée la plus abondante sur terre, l'eau est la ressource la plus cruciale pour toute activité humaine. C'est une ressource indispensable pour l'entretien vitale de toute vie et déterminante pour toute démarche de développement économique et sociale. c'est pourquoi sa gouvernance est considérée comme l'élément majeur lors de l'élaboration de toute stratégie même pour les plus pays développés. 
Cependant, l'eau n'a pas été bien considérée comme facteur de développement dans les pays sous-développés, même elle entrave la mise en œuvre de tout projet ou plan de développement, et malgré ça, nombre de décideurs ne lui affectent jamais un coefficient reflétant son importance, surtout que la majorité des études menées dans le domaine des ressources en eaux sont axées sur les aspects techniques plus que sur ceux économiques, sociologiques, ,sociologiques, juridiques ou politiques, la preuve on trouve plus d'hydrauliciens dans la production scientifique que les experts d'autres disciplines, puisqu'ils détiennent toutes les données concernant le secteur hydrique, c'est pourquoi d'ailleurs la littérature disponible est issue de technologues alors que les modèles d'analyses économiques sont presque insignifiants, on remarque aussi que la gestion de la ressource est souvent confiée aux technologues. C'est pourquoi l'évaluation de l'efficacité de tous les programmes n'est presque jamais prise en considération de manière pluridisciplinaire. Ce manque de vision et surtout dans son versant économique a aggravé la situation hydrique de plusieurs régions dans le monde, alors il est indispensable d'associer les diverses approches et de croiser les connaissanhydrauliques, hydrologiques et économiques ainsi que d'autres disciplines, ce qui va contribuer de façon synergique à l'amélioration et à la conservation de la ressource tout en valorisant son impact sur le développement socio-économique.

L'Algérie, à l'instar de tous les pays qui sont/seront affectés par la rareté de cette ressource, surtout en analysant sa situation à travers l'historique du secteur hydrique, est tenue d'adopter cette démarche pour éviter le pire.

Depuis l'occupation française, il a été constaté une répartition non-équilibrée de l'abondance de la ressource en eau entre les régions du pays notamment sa rareté et une pluviométrie insuffisante à l'Ouest du pays, c'est pourquoi il apparait dans les aménagements réalisés en 1920, sur 09 réservoirs, 06 étaient réservés aux seules plaines de l'Oranie et du Chélif.

La forte capacité de production agricole -notamment les céréales - en Algérie, a inspiré les occupants à créer un organisme pour la collecte des récoltes afin d'avoir un avantage compétitif lors des négociations sur les marchés internationaux, organe devenu après l'indépendance l'Office Algérien Interprofessionnel des Céréales, sachant que les céréales sont des produits nécessitant pour leurs production, d'énormes quantités d'eau, alors que le pays enregistrait des excédents destinés à l'exportation, ce qui prouve que l'Algérie disposait une disponibilité impressionnante d'eau. Pour renforcer cet avantage, plusieurs programme et travaux hydrauliques ont été entrepris, le dernier était bien avant $1962^{(1)}$ alors que ses résultats ont été enregistres après l'indépendance. 
A la vieille de l'indépendance, 14 barrages sont entrés en services en Algérie dont 10 dans la seule partie Ouest du pays. En ce qui concerne le déséquilibre pluviométrique qui existait entre des différentes régions, M. Côte (1996) observe que $93 \%$ de la capacité des barrages hérités de la période coloniale étaient localisées à l'ouest du méridien d'Alger.

\section{$\underline{\text { Analyse de la demande sur la ressource }}$}

Une nouvelle politique nationale s'installe alors, tout en visant le lancement de l'industrie industrialisante, une politique qui a favorisée le secteur industriel au détriment des autres secteurs notamment celui de l'agriculture et de l'hydraulique puisque en dix ans (1967-1977) la part d'investissement dans les deux secteurs est passée de $17,88 \%$ à $7,51 \%$ du budget total, alors que la demande était en permanente croissance, ce qui a engendré un retard dramatique et un déséquilibre entre les différents secteurs, puisque après ces mesures, les agriculteurs ont optés pour l'exode vers les villes et se sont installés dans les grandes usines, un secteur qui rapporte plus que l'agriculture.Il est à noter que sur les 0.45 Milliards de $\$^{(2)}$ alloués aux deux secteurs durant la même période (1967-1977) - prolongé jusqu'en 1979 -, seul 0.32 Milliards \$ ont été consommés, ce qui reflète l'inefficacité dans la gestion $\mathrm{du}$ secteur, alors que le budget alloué ne répondait pas aux vrais besoins du secteur, la situation s'aggrave davantage en consommant $71.11 \%$ de sa totalité. Les retombés de ce cumule ont été bien apparentes à partir des résultats enregistrés dès 1980 puisque l'objectif tracé par le secrétariat d'état à l'hydraulique en 1970 a été l'irrigation de 154000 ha à l'horizon 1980, alors que selon J.J.Pérennès, en 1980, une étude du Ministère du Plan de l'Aménagement du Territoire (MPAT) l'évaluait à 55000 ha, soit à peine 35,71 $\%$ des prévisions, un recul par rapport la superficie déjà irriguée en 1969 et le tableau suivant détaille l'évolution :

\section{Tableau (1)}

Unité : Superficie en Hectares

\begin{tabular}{|l|c|c|c|c|}
\hline & $\begin{array}{c}\text { Superficie } \\
\text { déjà } \\
\text { irriguée en } \\
1969\end{array}$ & $\begin{array}{c}\text { Nouveaux } \\
\text { objectifs à } \\
\text { réaliser entre } \\
1969 \text { et } 1980\end{array}$ & $\begin{array}{c}\text { Progression } \\
\text { attendu en } \\
1980\end{array}$ & $\begin{array}{c}\text { Total irrigué } \\
\text { à atteindre } \\
\text { en } 1980\end{array}$ \\
\hline $\begin{array}{l}\text { Grande } \\
\text { Hydraulique }\end{array}$ & 57000 & +97500 & $+171 \%$ & 154500 \\
\hline
\end{tabular}




\begin{tabular}{|l|c|c|c|c|}
\hline $\begin{array}{l}\text { Moyenne } \\
\text { Hydraulique }\end{array}$ & 140000 & +32500 & $+23,21 \%$ & 172500 \\
\hline $\begin{array}{l}\text { Petite } \\
\text { Hydraulique }\end{array}$ & 100000 & +24000 & $+24 \%$ & 124000 \\
\hline \multicolumn{1}{|c|}{ Total } & $\mathbf{2 9 7 0 0 0}$ & $+\mathbf{1 5 4 0 0 0}$ & $+\mathbf{5 1 , 8 5 \%}$ & $\mathbf{4 5 1 0 0 0}$ \\
\hline
\end{tabular}

Source :J.J.Pérennès, 1993, D'après Brahim BOUMAD : «Eau et développement durable en Algérie - Un enjeu important pour les populations et les territoires ", copy-merdia, Imprim'vert, France, Octobre 2010, p57.

Vu l'insuffisance du budget alloué aux secteurs, il était très difficile voire impossible d'atteindre les objectifs tracés, et ce malgré l'augmentation du ditbudget (qui est resté insuffisant) dans le deuxième plan quadriennal de 19741977, il est passé de 0.45 Milliards de \$ (1970-1973) à 1.092 Milliards de \$, soit une augmentation de $+142 \%$

Si on veut résumer le démarrage qui était censé participé au décollage de l'économie du pays conjointement avec l'industrialisation, on peut récapituler qu'en dix ans (1967-1977), seuls 1.14 Milliards de \$ ont été dépensés sur une enveloppe totale de 1.6285 Milliards de $\$$, soit $70,11 \%$, alors que les réalisations se résument essentiellement en :

- La construction de 03 barrages.

- L'estimation du volume régularisé de l'eau est passée de $250 \mathrm{hm}^{3}$ à $560 \mathrm{hm}^{3}$.

- 55000 ha seulement ont été équipés pour l'irrigation sur 166000 ha prévus (soit $33,13 \%$ )

Après ce bilan très alarmant, un intéressement a été attribué aux secteurs de l'agriculture et de l'eau afin de rattraper le retard qui a impacté la productivité agricole et par conséquent d'autres secteurs comme l'industrie agroalimentaire qui nécessitait d'énormes quantités d'approvisionnement en matière première, alors le budget passe de 1.092 à 2.8313 Milliards de $\$^{(3)}$ pour le premier plan quinquennal (1980-1984) avec de nouveaux objectifs notamment les grandes orientations pour l'achèvement des 26 barrages lancés pour un apport supplémentaire de $1200 \mathrm{hm}^{3}$.

De nombreuses décisions ont été prises dans le but de renforcer les capacités de maitrise, gestion et valorisation de la ressource, cette nouvelle éveil à fait naitre le comité de l'eau (1980) à caractère intersectoriel ${ }^{(4)}$ pour assurer les arbitrages dans l'affectation de l'eau et l'établissement d'un Plan Hydraulique National 
(PHN) en 1981 élaboré sur la base d'un découpage de l'Algérie du nord en 11 régions hydrauliques.

Après la crise de 1986 et la chute du prix de pétrole ce qui à remit en cause les capacités de financer et subventionner les activités liées au secteur, les autorités ont eu la certitude que le marché était instable et qu'ils auraient dû s'investir plus dans les secteurs les plus sures, à savoir l'agriculture, c'est pourquoi des crédits énormes ont été alloués au secteur avoisinant les 5.0471 Milliards de $\$$ (soit une augmentation de $+78 \%$ ) pour le deuxième plan quinquennal (19851989), ce chiffre est resté en permanente augmentation jusqu'à l'atteinte de 8.154 Milliards de $\$^{(5)}$ entre 2000 et 2004.

Pour bien évaluer l'impact de ces investissements surtout dans le secteur de l'agriculture sur le bien-être de la population notamment celle des régions rurales, on peut prendre quelques critères comme éléments indicateurs. En suivant l'évolution du ratio d'accès à l'eau potable en Algérie, on distingue rapidement son déclin, et même les prévisions menées ne donnent pas grand espoir, et ce comme suit :

\section{Figure (1)}

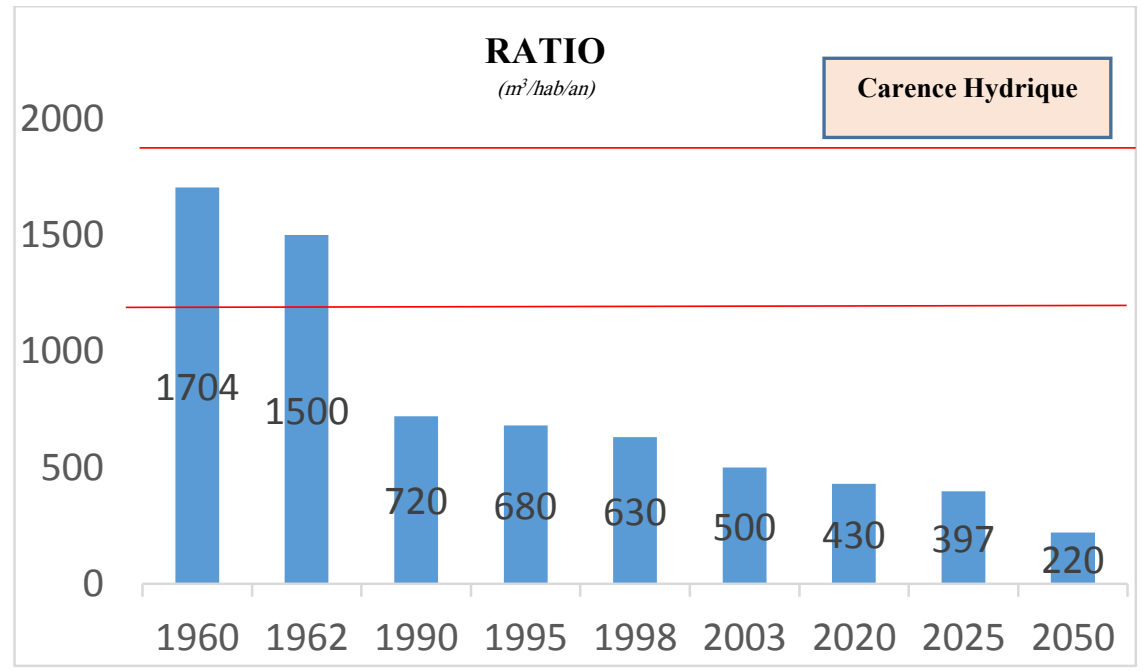

Source:https://www.wikipedia.org/ et http://donnees.banquemondiale.org/

Pour le cas de l'Algérie, on constate qu'elle était de justesse en Carence Hydrique en 1960 avant que ce taux commence à décliner, alors en 30 ans elle perd presque $1000 \mathrm{~m}^{3} / \mathrm{hab} / \mathrm{an}$, avant de franchir un seuil très critique et se 
retrouver en $500 \mathrm{~m}^{3} / \mathrm{hab} / \mathrm{an}$ depuis 2003, sans oublié que le taux est en permanent recule puisqu'il est prévu d'atteindre les $430 \mathrm{~m}^{3} / \mathrm{hab} / \mathrm{an}$ en 2020 , et $397 \mathrm{~m}^{3} / \mathrm{hab} / \mathrm{an}$ en 2025 , alors que seulement $220 \mathrm{~m}^{3} / \mathrm{hab} / \mathrm{an}$ en 2050 .

A noter que l'accès à une source d'eau améliorée est le pourcentage de la population qui a un accès raisonnable d'eau venant d'une source améliorée telle qu'une prise d'eau ménagère, un réservoir public au sol, un puits, une source ou un puit protégé ou eaux pluviales collectées. Les sources non améliorées comprennent les vendeurs, les camions citernes et les puits non protégés. L'accès raisonnable est défini étant la disponibilité quotidienne d'au moins 20 litres par personnes venant d'une source située dans un rayon d'un kilomètre de l'habitation. Dans ce contexte, l'Algérie est en recule vu son pourcentage qui s'est décliné de $94 \%$ en 1990 à $84 \%$ en 2014 surtout dès 2005 où le pays a enregistré un taux inférieur à celui de la moyenne mondial comme le montre la figure (2).

\section{Figure (2)}

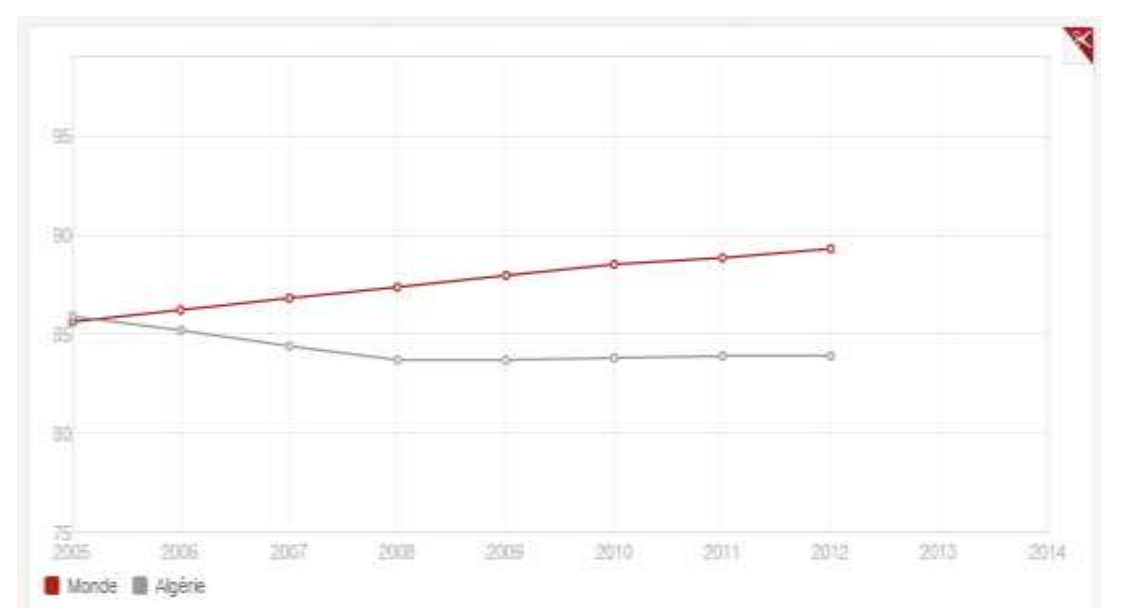

Source :http://donnees.banquemondiale.org/indicateur/SH.H2O.SAFE.ZS/cou ntries/1W-DZ?display=graph 


\section{Figure (3)}

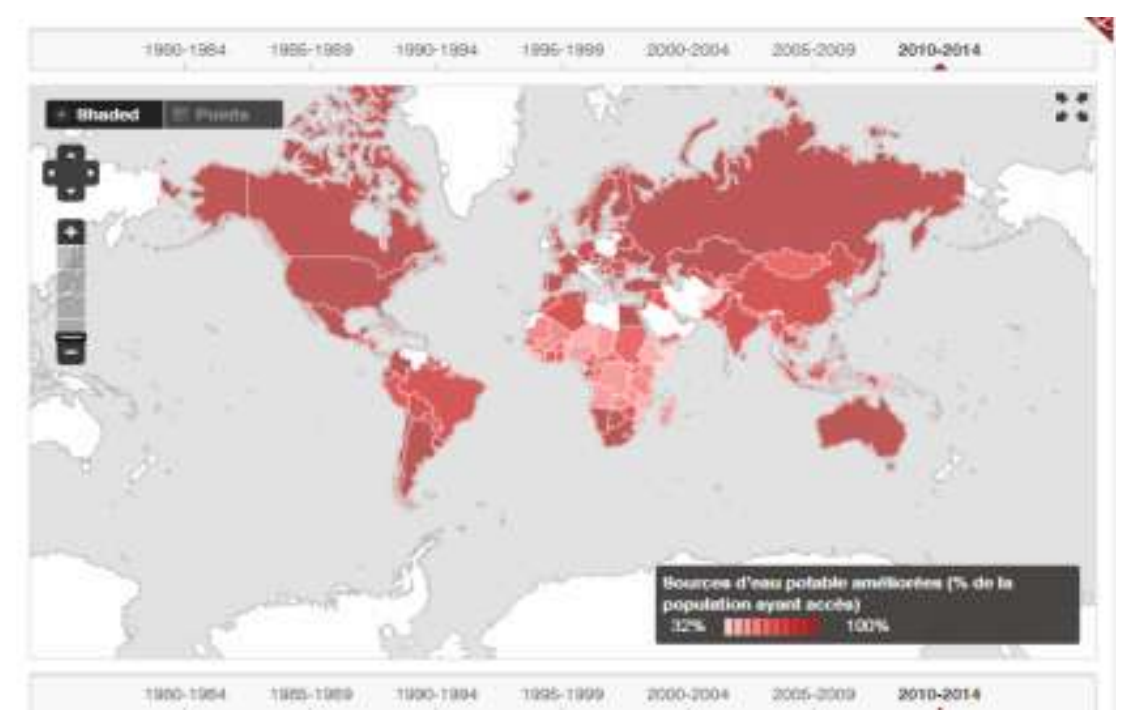

Source:

http://donnees.banquemondiale.org/indicateur/SH.H2O.SAFE.ZS/countries/1W -DZ? display=map

C'est pourquoi l'Algérie est classée comme pays à haut risque de crise, voire pénurie hydrique puisque ses ratios sont en perpétuelle déclinaison malgré le taux actuel qui est très élevé (figure 3), mais la tendance future en recule permanent n'est pas un indicateur prometteur.

Afin de trouver une explication à ce problème devenu parmi les causes qui freinent le développement local et/ou régional, la croissance urbaine galopante est l'une des plus importantes, on présente l'évolution de la population durant presque la même période comme suit : 


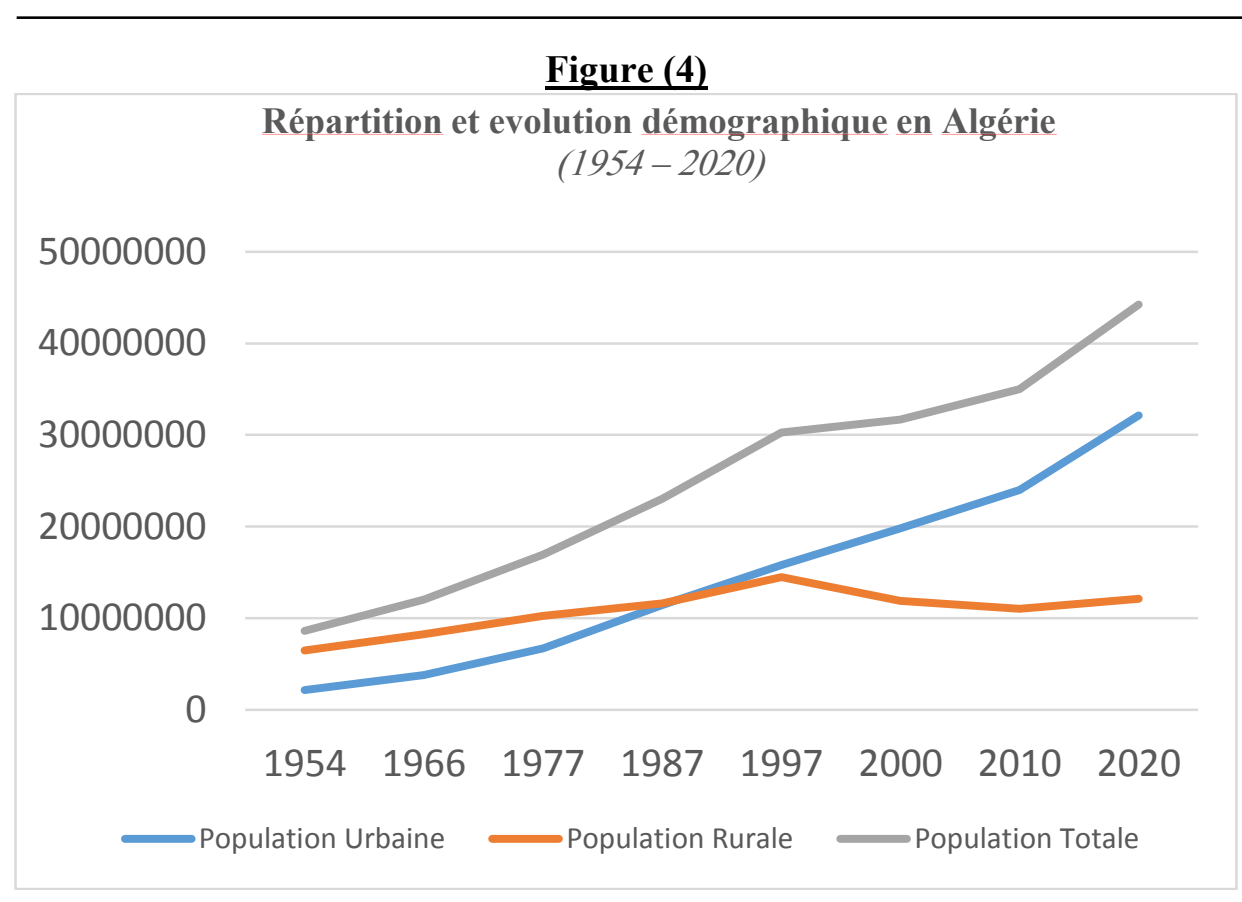

Source : Données de l'Office National des Statistiques, Alger.

***prévisions du Conseil National Economiques et Sociales (CNES), Alger 2004.

Selon les graphes de la figure (4), il est claire que la croissance de la population globale était à forte proportion en population urbaine en plus du recule de la population rurale à partir de 1997, cette forte concentration de la population engendre une hausse des besoins urbains, notamment au Nord du pays où se trouve $85-90 \%$ de la population totale, cet encombrement et concentration rendent la demande sur la ressource en eau très grande ainsi que la gestion des eaux usées qui lui est subséquente. C'est pourquoi cette demande excédentaire est le défi qui doit être démarketé correctement et intelligemment ${ }^{(6)}$.

L'urbanisation nécessite un lourd investissement dans les structures de base, mais en termes d'agglomération, la forte densité (surtout au Nord) implique des soucis d'approvisionnement en eau potable et un système de gestion très efficace pour maitriser les eaux usées issues de l'usage exponentiel au quotidien de cette ressource, chose qui n'a pas été assurée convenablement durant toute la période présentée. 
Malgré cette forte hausse de la population urbaine, il reste -paradoxalementque la population rurale la plus importante en consommation puisque dans les pays arabes, le secteur agricole consomme d'énormes quantités d'eau disponible alors que la contribution de toute l'hydraulique (petite, moyenne et grande) est minime au $\mathrm{PIB}^{(7)}$, alors que le reste se répartit entre les autres secteurs avec une dotation globale journalière en consommation domestique $(55 \%)$, industrielle $(15 \%)$, municipale $(10 \%)$ et les pertes $(20 \%)^{(8)}$.

Convaincu par tous les indices que l'agriculture est le noyau de la question hydrique dans le pays, on a voulu décortiquer le secteur pour bien identifier l'imperfection surtout que l'agriculture irriguée consomme environ $90 \%$ des ressources en eau disponibles de la région (Moyen Orient \& Afrique de Nord) et contribue à un pourcentage qui se situe entre 5 et $20 \%$ du PIB, selon le pays $^{(9)}$.

Pour la superficie irriguée, on enregistre :

Figure (5)

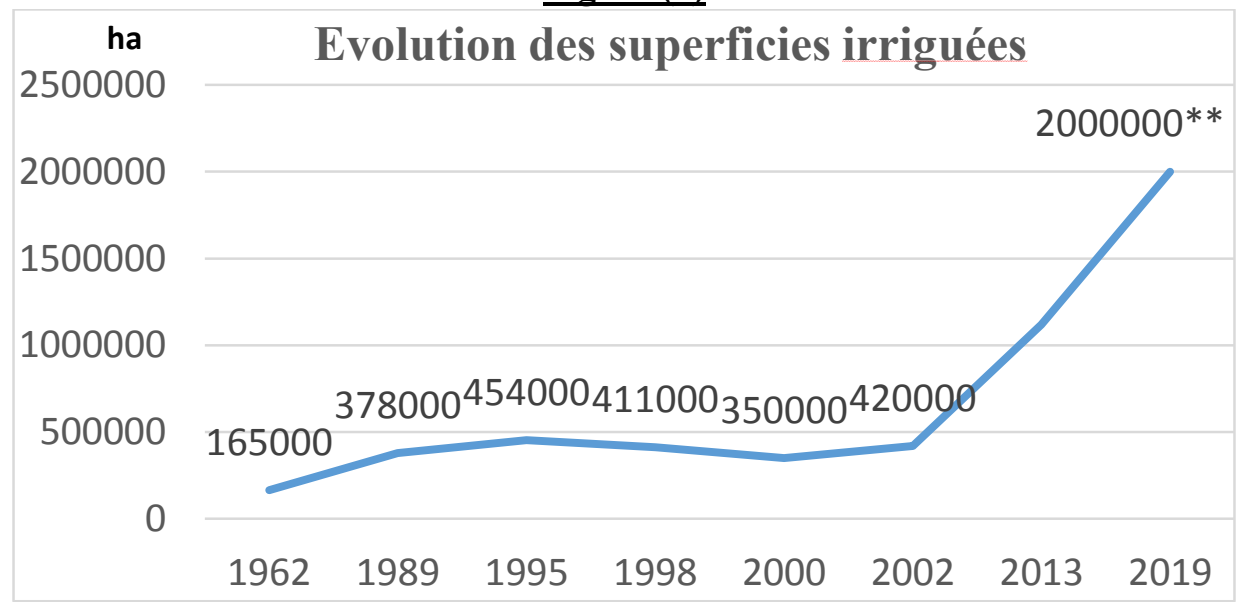

Sources : les chercheurs en se basant sur plusieurs données collectées.

** :Mohamed KESSIRA: "Programme de l'Economie de l'eau : Objectifs et Impacts », Document interne au MINISTERE DE L'AGRICULTURE ET DU DEVELOPPEMENT RURAL, Direction de développement agricole dans les zones arides et semi-arides. 
Les superficies irriguées n'étant que de 350000 ha en l'an 2000 pour passer à 1 119259 ha à fin 2013, selon le programme élaboré par le ministère de l'agriculture, l'objectif d'irriguer 2 Millions

d'hectares d'ici $2019^{(10)}$ a été fixé, mais pour une dose moyenne de 6000 $\mathrm{m}^{3} / \mathrm{ha} / \mathrm{an}$, il faudra mobiliser 12 Milliards de $\mathrm{m}^{3}$ à partir des eaux conventionnelles et non-conventionnelles et ce pour des fins de modernisation.

On admet généralement que la consommation d'un hectare de culture irriguée est de $5000 \mathrm{~m}^{3} /$ an dans le Tell, de 8000 en zone steppique, de 10 à 20000 au Sahara $^{(11)}$, et c'est pourquoi la moyenne de $6000 \mathrm{~m}^{3} / \mathrm{ha} /$ an a été adoptée pour évaluer les besoins théoriquement nécessaires pour atteindre l'objectif de superficie à irriguer en 2019.

Selon la figure (5), il est flagrant que l'objectif est abusivement optimiste, surtout en sachant que l'exode rural ne cesse de s'accélérer et les dispositifs misent en ouvre par l'Etat pour encourager les agriculteurs à maintenir leurs activités ne fonctionnent pas de la même manière que la stratégie élaborée depuis des décennies. L'argent est souvent détourné ou investi dans des créneaux plus porteurs que l'agriculture tels que l'importation et le commerce.

Aussi la surexploitation de la ressource au seul secteur de l'agriculture ne permet pas d'espérer d'atteindre un tel objectif puisque théoriquement l'irrigation de 2 Millions d'hectares nécessite la mobilisation de 12 Milliards de $\mathrm{m}^{3} / \mathrm{an}$ à raison de $6000 \mathrm{~m}^{3} / \mathrm{ha} / \mathrm{an}$ alors que la stratégie de la grande Hydraulique vise d'atteindre une capacité de mobilisation de la ressource de 8,4 Milliards de $\mathrm{m}^{3} /$ an avec le plan de construction de 84 barrages, alors un déficit théorique de 3.6 Milliards de $\mathrm{m}^{3} /$ an vient d'être identifié.

En plus de ce petit calcul, vient de s'ajouter l'écart qui existe entre les données théoriques et les informations collectées par les différentes études menées, puisque même les capacités des barrages ne peuvent pas être atteintes tant que le taux de remplissage est asymétrique (Figure 6) et qui est entre 10 et $32 \%$, ce qui élargie la mosaïques des arguments qui mettent cet objectif en cause et le rendent irréaliste, en plus de l'envasement qui affecte la plupart des barrages. une étude menée par B.Remini ${ }^{(12)}$ a conclue qu'un volume de 32 Millions de $\mathrm{m}^{3}$ de vase (en 2002) s'ajoute annuellement dans les 52 barrages (sujet de l'étude), le volume de vase était estimé de 1 Milliard de $\mathrm{m}^{3}$ en 2004, la quantité prévue en 2010 était de 1.1 Milliards de $\mathrm{m}^{3}$ et de 1.35 milliard de $\mathrm{m}^{3}$ en 2020 pour le même nombre de barrage. 


\section{Figure (6)}

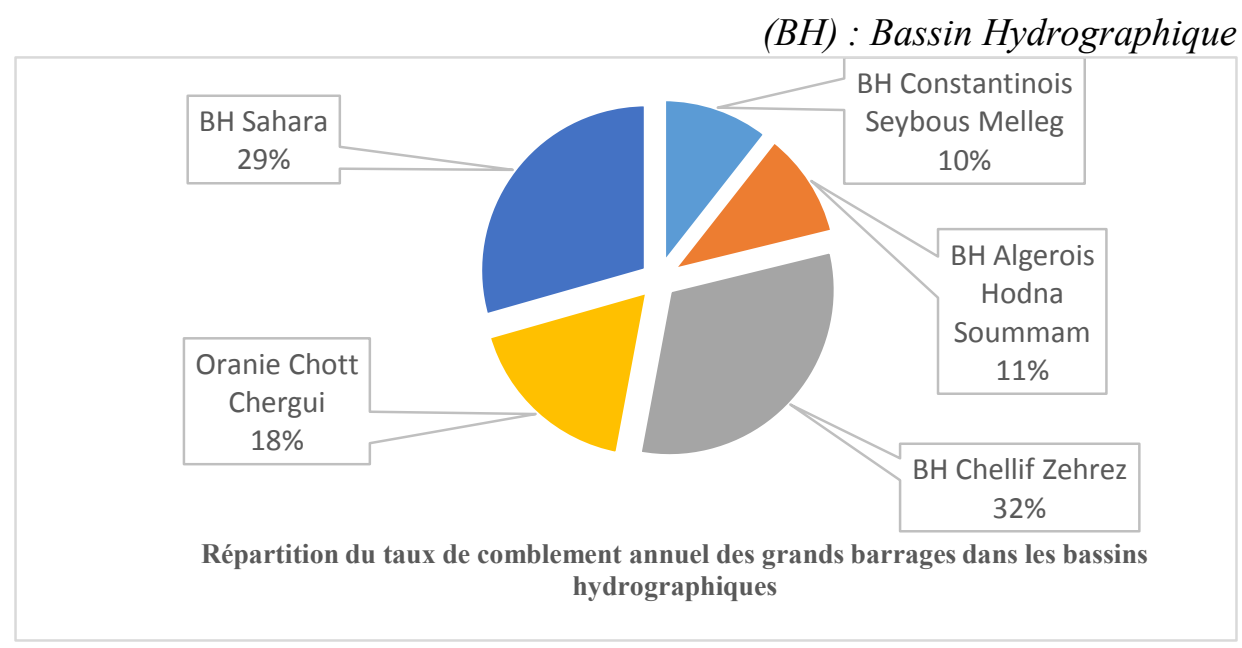

Source : REMINI B., HALLOUCHE W : «Les barrages du Maghreb face au phénomène de l'envasement », Revue VECTEUR Environnement (Canada). Novembre, Vol 36 no 6, 2003, pp. 27-30.

Pour la subvention de l'eau agricole ${ }^{(13)}$, on enregistre un taux de $66 \%$ en Algérie en comparaison avec les USA 49\% et au Japon qui est de l'ordre de $30 \%$, mais malgré cette forte subvention, l'accès informel à la ressource y est largement par des forages illicites et même en recourant à l'irrigation avec l'eau des Oueds sans aucune autorisation ou procédure légale, car les agriculteurs optent pour l'acquisition de la ressource gratuitement que de payer une eau (subventionnée ou pas). 


\section{$\underline{\text { Conclusion }}$}

Les programmes élaborés et réalisés pour une future mise en œuvre ne répondent pas aux normes et exigences technico-économiques tant que le retour sur investissement n'est pas garanti et le ratio d'accès à l'eau est en rapide déclin et s'accélère avec les problèmes liés à la ressource notamment le déséquilibre en dotation entre les différents secteurs, les réseaux vétustes d'accès à l'eau, une population galopante et le gaspillage causé et aggravé par l'usage de techniques inappropriées.

En plus de ce qui précède, des objectifs irréalistes sont fixés lors de l'élaboration des programme d'économie de l'eau et de modernisation, sous forme de décision unilatérale, alors que l'historique nous renseigne que objectifs restent des visions utopiques et qu'elles ne seront misent en œuvre qu'en allouant les sommes conséquentes, sans que cela puisse autant garantir l'efficacité de ces programmes et leurs impact sur la sphère socio-économique et/ou sociétale. Aussi cessommes ne seront pas utiles tant que la gouvernance n'est pas assurée avec une stratégie efficace afin d'être plus réaliste et pouvoir atteindre les objectifs ciblés.

Une nouvelle réflexion devrait être instaurée pour le secteur car la délinquance est impardonnable dans les questionnements vitaux, surtout qu'il est confié dans la plupart des cas aux dirigeants qui croulent sous les tâches administratives et souvent n'ont pas les compétences requises dans ce domaine o combien exigeant.

\section{Les Marges :}

(1)05 Juillet 1962 : date qui signifie l'indépendance de L'Algérie après 132 d'occupation par la France.

(2)1 $\mathrm{DZD}=0.2374 \$$ (moyenne de taux de change de la période 1962-1980, calculé sur http://fxtop.com/fr/historique-taux-change.php).

(3)1 DZD $=0.1231 \$$ (moyenne de taux de change de la période 1980-1998, calculé sur http://fxtop.com/fr/historique-taux-change.php).

(4)Le secteur de l'eau était affilié durant les plans précédents à différentes institutions de tutelles avant l'instauration d'une politique claire sous la tutelle du Ministère de l'Hydraulique en 1980. 
(5)1 DZD $=0.0135 \$$ (moyenne de taux de change de la période 1999-2014, calculé sur http://fxtop.com/fr/historique-taux-change.php).

(6)Mohamed Sofiane CHAOUI \&Azzedine BENTERKI: « The Demarketing: Effective Strategy to rationalize Water Consumption ", LJEE, $n^{\circ} 23$, December 2013, p7.

(7)PIB:Produit Intérieur Brut.

(8)Brahim BOUMAD : «Eau et développement durable en Algérie : Un enjeu important pour les populations et les territoires », copy-merdia, Imprim'vert, France, Octobre 2010, p69.

(9)Serren JUMA : « La gestion des ressources en eau au Moyen Orient et en Afrique du Nord », Note Sectorielle, The World Bank, Washington, Août 2003.

(10) Mohamed KESSIRA: "Programme de l'Economie de l'eau: Objectifs et Impacts", Document interne au Ministère de l'Agriculture et du Développement Rural, Direction de développement agricole dans les zones arides et semi-arides, Février 2015.

(11) Azzedine MEBARKI: "Le défi de l'eau en Algérie : Aménagements régionaux et gestion de la ressource ", Rencontre internationale: Gestion Durable de l'eau en Méditerranée » Université Mentouri de Constantine, Ambassade de France, Agence de Bassin Hydrographique C.S.M, Constantine, Algérie, 19-20 Mars 2007, p 4.

(12) Boualem REMINI, «La problématique de l'eau en Algérie du Nord», Larhyss Journal, n8, Juin 2010, p36.

(13) Lakhdar Zella \& D. SMADHI : «Gestion de l'eau dans les pays arabes », Larhyss Journal, Laboratoire de Recherche en Hydraulique Souterraine et de Surface, n05, Biskra, Algérie, Juin 2006, p10. 


\section{Références}

- Azzedine MEBARKI, 19-20 Mars 2007 : «Le défi de l'eau en Algérie : Aménagements régionaux et gestion de la ressource», Rencontre internationale: Gestion Durable de l'eau en Méditerranée " Université Mentouri de Constantine, Ambassade de France, Agence de Bassin Hydrographique C.S.M, Constantine, Algérie.

- Boualem REMINI, Juin 2010 : «La problématique de l'eau en Algérie du Nord $»$, Larhyss Journal, $n^{\circ} 8,27-46$.

- Brahim BOUMAD, Octobre 2010: «Eau et développement durable en Algérie: Un enjeu important pour les populations et les territoires", copy-merdia, Imprim'vert, France.

- Conseil National Economiques et Sociales (CNES), Alger.

- J.J.PERENNES, 1993 : L'eau et les hommes au Maghreb, Contribution à une politique de l'eau en Méditerranée ", Kharthala, Paris.

- Lakhdar ZELLA \& D. SMADHI, Juin 2006: «Gestion de l'eau dans les pays arabes », Larhyss Journal, « Laboratoire de Recherche en Hydraulique Souterraine et de Surface », n05, Biskra, Algérie.

- Ministère de l'équipement et de l'aménagement du territoire "Conférence Nationale sur la nouvelle politique de l'eau», acte de conférence nationale, AGEP, Janvier 1995.

- Ministère des Ressources en eau : Documents internes, Avril 2015.

- Mohamed KESSIRA, Février 2015: "Programme de l'Economie de l'eau: Objectifs et Impacts », Document interne au Ministère de l'Agriculture et du Développement Rural, Direction de développement agricole dans les zones arides et semi-arides.

- Mohamed Sofiane CHAOUI \&Azzedine BENTERKI, December 2013: «The Demarketing: Effective Strategy to rationalize Water Consumption », LJEE, n²3, 5-20.

- REMINI B., HALLOUCHE W, 2003: «Les barrages du Maghreb face au phénomène de l'envasement», Revue VECTEUR Environnement (Canada). Novembre, Vol $36 \mathrm{n}^{\circ} 6$.

- SerrenJuma, Août 2003 «La gestion des ressources en eau au Moyen Orient et en Afrique du Nord», Note Sectorielle, The World Bank, Washington, USA.

- $\quad$ Site Web :http://www.abhs.dz/php/fr/agence.php

- Site Web: www.ons.dz 
- Site Web: www.donnees.banquemondiale.org

- Site Web: www.mre.dz

- Site Web: www.wikipedia.org 